\title{
IMPROVING THE SHELF LIFE AND QUALITY OF CUT GLADIOLUS CV. "WHITE PROSPERITY" SPIKES BY ETHYLENE INHIBITOR, COLD STORAGE AND THEIR INTERACTIONS
}

\author{
Azza M. Abdel-Moniem \\ Floriculture Res. Dept., Hort. Res. Inst. ARC, Giza, Egypt
}

Received: May 18, 2016

Accepted: Jul. 31, 2016

\begin{abstract}
The study was conducted at the Postharvest Lab. of Floriculture Res. Dept., Hort. Res. Inst., Giza, Egypt during 2012 and 2013 seasons to explore the effect of silver thiosulphate solution (STS) applied as foliar spray or as immersing solution for 10, 20 and 30 minutes (control spikes were immersed in distilled water), cold storage at $5{ }^{\circ} \mathrm{C}$, vertically or horizontally for 2 or 4 days, beside control treatment in which spikes were kept at room temperature and their interactions on water relations, longevity and quality of Gladiolus hybridus cv. "White Prosperity" cut spikes. After each treatment, the bases of spike stems were held in $10 \%$ sucrose solution till the end of the experiment.

The obtained results showed that STS pulsing treatments caused a significant increment in the amount of water uptaken by cut Gladiolus spikes, and significantly decreased, the amount of water loss compared to control in most cases of the two seasons. These treatments were also improved floret diameter, vase life, spike fresh and dry weights, dry weight \%, opening $\%$, leaf content of chlorophyll $a, b$ and reducing sugars \% in the leaves petals, and decreased wilting \%, and carotenoids content in the leaves and total sugars \% in the leaves and petals. However, the prevalence was for immersing in STS solution for 10 min treatment, which gave the best values in most previous characters with few exceptions in both seasons. Storage at room temperature or at $5^{\circ} \mathrm{C}$, vertically or horizontally for 2 or 4 days alternated improving means of previous characters with the mastery of horizontal cold storage treatment for 2 days, which recorded the best results in most cases. The interaction treatments also induced a marked improvement in most measurements, but the dominance was for the combining between immersing in STS solution for $10 \mathrm{~min}$. and horizontal cold storage for 2 days, as this combination gave the best water relations, the longest vase life and the highest quality compared to the other individual and combined treatments.

Accordingly, it is recommended to immerse the cut spikes of Gladiolus cv. "White Prosperity" in STS solution for $10 \mathrm{~min}$ and then holding them in $10 \%$ sucrose solution at room temperature for local marketing or storage them after immersing in STS solution, horizontally for at least 2 days at $5^{\circ} \mathrm{C}$ for shipment abroad.
\end{abstract}

Key words: Gladiolus, shelf life, STS, cold storage, Postharvest treatments.

\section{INTRODUCTION}

Gladiolus L., Corn flag or Sword lily that belongs to Fam. Iridaceae is one of the four famous commercial cut flowers in the world (Bai et al., 2009). However, the smaller flowered cultivars are used for the herbaceous border. It is a tender and perennial herb with tunicate corms. Native to Europe, Mediterranean region, the Near East, but chiefly to tropic and South Africa. Flowers are showy, sometimes fragrant, in 1 -sided spikes. Propagated mainly by corms and cormels (Bailey, 1976).
The longevity of gladiolus cut flowers is very short. The typical vase life of individual florets is just 4 to 6 days (Yamada et al., 2003). Therefore, many efforts were done to increase the longevity of this important cut flower, such as those elicited by Rekha and Shankaraiah (2002) who found that an increase in vase life and fresh weight of cut gladiolus spikes was significantly more with spikes held in $\mathrm{PM}+3 \%$ sucrose $+200 \mathrm{ppm}$ 8-HQS solution for 22 days at cold storage $\left(5{ }^{\circ} \mathrm{C}\right)$ conditions. Sashikalal and Ranvir (2003) noticed that both cellophane and 
butter paper packed flowers stored at $4{ }^{\circ} \mathrm{C}$ for 2 days, in cellophane or butter paper for 4 days and in butter paper for 6 days proved to be the best to increase the postharvest life of Gladiolus cv. Her Majesty cut spikes. On gladiolus cut spikes cv. Peater Pears, Singh et al., (2007) reported that cold storage at $6-10{ }^{\circ} \mathrm{C}$ for 10 days with polypropylene $(60 \quad \mu)$ film packaging maintained good keeping quality with improved floret opening, floret size and petal colour.

Similar observations were also detected on gladiolus cut spikes by Beura et al., (2001), Hassan (2005), Singh et al., (2009), Marandi et al., (2011) and Shimizu-Yumoto and Ichimura (2015) whom noted that pretreatment with STS improved bud opening and longevity of cut gladiolus "Princess Summer Yellow" spikes after suboptimal temperature storage for 6 days.

On other cut flowers, several reports were also recorded by Hutchinson et al., (2003) on tuberose, Jul et al., (2007) on amaryllis, Solgi et al., (2009) on gerbera, Gendy and Hamad (2011) on Strelitzia reginae, Shahri et al., (2011) on cut spikes of Consolida ajacis cv. Violet Blue, Bayleyegn et al., (2012) on rose, Santos et al., (2012) on Epidendrum ibaguense, Abdel-Moniem et al., (2012) on Rosa hybrida cvs. Anna, Gold Strike and Spot and Abd-Allah et al., (2013) on Asiatic hybrid lily cv. Orange Tycoon.

Regarding the effect of storage method, Muchiri (2005) mentioned that horizontal packaging of Eustoma grandiflorum cut flowers at $2{ }^{\circ} \mathrm{C}$ caused geotropic bending of flower pedicels. Cut inflorescences stored horizontally in boxes had their flower pedicels remaining bent not less than $15^{\circ}$ throughout the display period, whereas those stored vertically in boxes had no detectable geotropic curvature. Longer periods of horizontal packaging progressively increased geotropic curvature of flower pedicles and this in effect reduced the overall postharvest quality of cut flowers.
Moreover, Celikel et al., (2010) pointed out that when cut spikes of snapdragon were placed horizontally at $20^{\circ} \mathrm{C}$, growth became negatively gravitropic within $20 \mathrm{~min}$. Bending was significantly higher than control (stored vertically) in all flowers stored horizontally at temperatures above $5^{\circ} \mathrm{C}$.

Analogous results were also explored by Philosoph-Hodas et al., (1996) on snapdragon, Han (2001) on lilies and Celikel and Reid (2002) and Watkins and Miller (2005) on gerbera and sunflower and indicated that the best storage temperature for gladiolus spikes (as buds) ranged between $4-6{ }^{\circ} \mathrm{C}$ for $5-8$ days proved keeping them in vertical position.

The purpose of this trial is to explore the most suitable pulsing treatment and storage method reliable for increasing longevity and freshness of cut gladiolus spikes cv. White Prosperity.

\section{MATERIALS AND METHODS}

The current work was carried out at the Postharvest Lab of Floriculture Res. Dept., Hort. Res. Inst., Giza, Egypt during the two consecutive seasons of 2012 and 2013 to examine the effect of silver thiosulphate, cold storage and their interactions on longevity, water relations and quality of one of the commercial cultivar of gladiolus.

Fresh cut spikes of Gladiolus hybridus cv. "White Prosperity" were obtained on January, $16^{\text {th }}$ for each season from a local commercial farm (Floramax, El-Mansouria, Giza), picked in the early morning when the first flower bud showed the full colour (export stage) at similar lengths of $60 \mathrm{~cm}$ and were directly divided into groups and wrapped inside kraft paper and transported as quick as possible to the laboratory. Once in the Lab., the cut spikes were firstly precooled by immersion in a cool water (about $7{ }^{\circ} \mathrm{C}$ ) for $30 \mathrm{~min}$. to remove the field heat. Subsequently, stem bases were recut under water by removing about $1 \mathrm{~cm}$ and the cut spikes were divided into similar and equal five groups, as each one of them received one of the following treatments: 
1. The treatment with silver thiosulphate (STS) solution, prepared by dissolving $0.34 \mathrm{~g} \mathrm{AgNO}_{3}$ in $125 \mathrm{ml}$ distilled water (solution A) and $0.632 \mathrm{~g}$ sodium thiosulphate in $125 \mathrm{ml}$ distilled water (solution B). Solution A was slowly added to solution B with shaking to get $250 \mathrm{ml}$ of STS solution (Reid et al., 1980 b).

The solution of STS was used as: spray on the foliage of the cut spikes, and as pulsing solution where the bases of spike stems were immersed in it for 10,20 or 30 min., beside control treatment in which the stem bases were immersed in distilled water. This group was kept at room conditions (as the temperature, $\mathrm{RH}$ and light intensity during the course of study ranged between; $18 \pm 2{ }^{\circ} \mathrm{C}, 45 \pm 5 \%$ and $900-1000$ lux, respectively) and referred to as pulsing or Lab treatments.

2. Each one of the remained 4 groups was subjected to the previously mentioned lab treatments plus cold storage at $5{ }^{\circ} \mathrm{C}$, vertically or horizontally for either 2 or 4 days to form the following 4 cold storage treatments:

- Storage for 2 days, vertically.

- Storage for 2 days, horizontally.

- Storage for 4 days, vertically.

- Storage for 4 days, horizontally.

3. The interaction treatments: each treatment of pulsing (lab) treatments was combined with each one of cold storage treatments to create 25 interaction treatments.

At the end of as each one of the aforenamed treatments, the bases of spike stems were hold in clear glass jars (3 spikes/jar) containing about $200 \mathrm{ml}$ of $10 \%$ sucrose solution till the end of the experiment. A drop of xylene was added on the surface of sucrose solution to prevent evaporation during the vase life period. The jars with spikes inside were kept at room temperature $\left(18 \pm 2{ }^{\circ} \mathrm{C}\right)$ under normal daylight and natural ventilation. The lay out of the experiment in the two seasons was a complete randomized blocks in factorial experimental type, replicated thrice as each replicate included 3 spikes (Mead et al., 1993).

Data were recorded as follows: water uptake and water loss (g/spike) were evaluated during the shelf period once every 3 days and recorded in the tables as the mean for the whole shelf period. Water balance was calculated by subtracting water loss mean from water uptake mean, floret diameter (cm), vase life (day), spike fresh and dry weights (g), dry weight \% using the equation of: DW \% = dry weight/fresh weight $x$ 100, as well as opening and wilting percentages. In ethanolic extracts, the content of chlorophyll $\mathrm{a}, \mathrm{b}$ and carotenoids (mg/g f.w.) in fresh leaf samples, total and reducing sugars percentages in fresh leaf and petal samples were determined according to the methods of Moran (1982) and Dubois et al., (1966), respectively.

Data were then tabulated and statistically analysed using SAS Institute Program (1994). Differences among treatments were compared by Duncan's New Multiple Range Test (Steel and Torrie, 1980).

\section{RESULTS AND DISCUSSION Effect of silver thiosulphate, cold storage treatments and their interactions on: \\ 1- Water relations of cut spikes.}

It is obvious from data averaged in Table (1) that amount of water uptake by gladiolus cut spikes increased significantly by STS spraying or pulsing treatments compared to control treatment (D.W.) in the two seasons. The opposite was the right concerning amount of water loss, which decreased significantly by the same treatments relative to control treatment in both seasons. However, the highest amount of water uptake coupled with the least one of water loss was achieved in the two seasons by pulsing in STS solution for $10 \mathrm{~min}$. It could be said that, the best water balance was achieved in the two seasons by this treatment which raised such parameter to 33.47 and $30.58 \mathrm{~g}$ in the first and second seasons, respectively. 


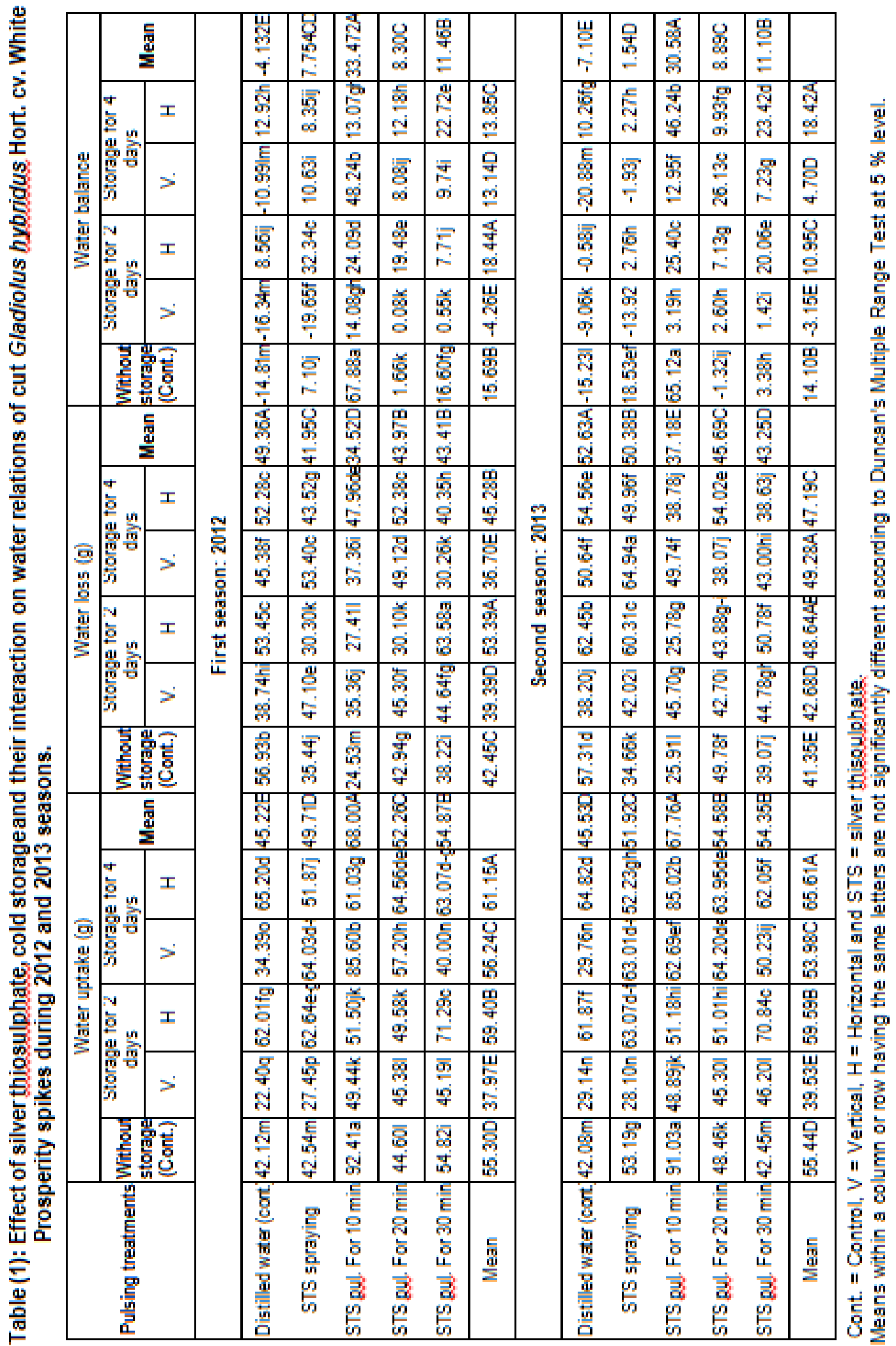


As for the effect of storage treatments, it was noticed that cold storage for 4 days increased water uptake to the highest amount when spikes were kept horizontally compared with the other treatments in both seasons, while the least amount of water loss was attained in the first season by storing the spikes vertically for 4 days treatment, followed by vertical storage treatment for only 2 days. In the second season, that was attained by the control treatment (storage at room temperature), which reduced water loss value to $41.35 \mathrm{~g}$ and followed by vertical storage treatment for only 2 days, that decreased the mean of such parameter to $42.68 \mathrm{~g}$, compared to the other treatments.

On the other hand, the interaction treatments caused variable effects on water relations of gladiolus cut spikes. The best water relations registered in the two seasons was attributed to the combining between pulsing spikes in STS solution for $10 \mathrm{~min}$. and keeping them at the room temperature, as this combination raised water uptake to the highest value, decreased the water loss to the minimal value. Consequently the best water balance means $\left(67.88 \mathrm{~g}\right.$ in the $1^{\text {st }}$ season and $65.12 \mathrm{~g}$ in the $2^{\text {nd }}$ one) with highly significant differences over all the other combinations in the two seasons. This may be attributed to that STS inhibited the action of ethylene and lead to a decrease in lip oxygenase (Lox) activity, as well as served as an antibacterial component, beside that all vital processes, including photosynthesis are well carried out under room temperature, and this finally will maintain the water balance of spikes by regulating the water flux into the xylem vessels and controlling transpiration (Han, 2001).

These results are in accordance with those revealed by Beura et al., (2001), Hassan (2005), Singh et al., (2009) and Shimizu-Yumoto and Ichimura (2015) on gladiolus. In this connection, Marandi et al., (2011) claimed that STS treatment at 150 ppm had positive effect on water uptake by gladiolus cut spikes. Gendy and Hamad (2011) found that pulsing Bird-of-Paradise cut flowers in STS solution (1:4 mM) for 30 min., then in solution containing $20 \%$ SUC + $200 \mathrm{ppm}$ 8-HQS for $12 \mathrm{~h}$ significantly improved water balance and maintained flower quality. When the previous pulsing treatment interacted with cold storage for 5 days at $6 \pm 1^{\circ} \mathrm{C}$, the highest water balance and quality were obtained. Abd-Allah et al., (2013) stated that storage under room temperature recorded the highest amount of water uptaken by lily flowering stems compared with cold storage treatments. Storage at $2{ }^{\circ} \mathrm{C}$ gave the least means in this parameter. The inflorescences rapidly wilted after the ninth day from cut. This may be due to increasing petal sensitivity after cold storage, depletion of carbohydrate reserves and oxidative stress caused by cold storage.

\section{2- Floret diameter and vase life:}

Data in Table (2) clear that floret diameter and vase life increased significantly in response to the different pulsing treatments used in this trial. However, the superiority was for pulsing in STS solution for $10 \mathrm{~min}$. treatment which gave the highest records in both seasons over the control and other pulsing treatments. This may indicate the role of STS as ethylene binding inhibitor and as a biocide, so improves the vase life of cut flowers by delaying its senescence (AbdelMoniem et al., 2012). On the other side, storage at room temperature greatly improved floret diameter in the two seasons, whereas cold horizontal storage for 4 days significantly improved this character in the second season only. Vase life, however extended markedly by storage at room temperature and cold horizontal storage for 2 days treatments in the $1^{\text {st }}$ season, while in the $2^{\text {nd }}$ one, the longest vase life was established by cold horizontal storage for 2 days and cold vertical storage for 4 days treatments. This may be reasonable because all vital processes, including 


\begin{tabular}{|c|c|c|c|c|c|c|c|c|c|c|c|c|c|c|c|c|c|}
\hline & & & & $\begin{array}{l}8 \\
8 \\
06 \\
0\end{array}$ & $\begin{array}{l}\text { 思 } \\
\text { 今్ } \\
\text { g. }\end{array}$ & 咅 & $\begin{array}{l}\text { 畹 } \\
\text { ó }\end{array}$ & 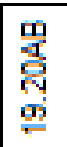 & & & & 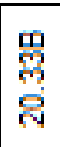 & 営 & 㗖 & 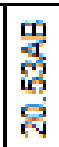 & & \\
\hline & $\frac{5}{0}$ & $I$ & & $\begin{array}{l}8 \\
8 \\
0 \\
0\end{array}$ & 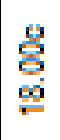 & 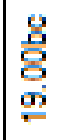 & $\begin{array}{l}8 \\
8 \\
0 \\
0\end{array}$ & $\begin{array}{l}8 \\
8 \\
\text { ó }\end{array}$ & 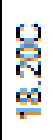 & & 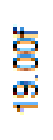 & 总 & 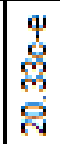 & 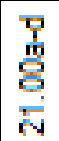 & 莺 & 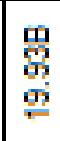 & \\
\hline 鸽 & कू & $>$ & & $\begin{array}{l}8 \\
8 \\
00 \\
0\end{array}$ & $\begin{array}{l}8 \\
8 \\
8 \\
0\end{array}$ & $\begin{array}{l}8 \\
8 \\
\\
\\
\end{array}$ & $\begin{array}{l}8 \\
8 \\
\text { ó }\end{array}$ & 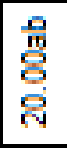 & $\begin{array}{l}\text { 䍛 } \\
\text { क् }\end{array}$ & & 类 & 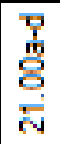 & 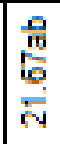 & $\begin{array}{l}\frac{0}{0} \\
\frac{\pi}{6} \\
\frac{\bar{N}}{\mathrm{~N}}\end{array}$ & 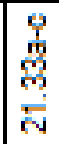 & 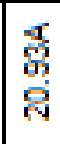 & \\
\hline 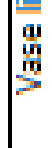 & 哀 & $I$ & & $\begin{array}{l}8 \\
8 \\
0 \\
0\end{array}$ & $\begin{array}{l}\text { 용 } \\
\text { 옹 }\end{array}$ & $\frac{\mathrm{m}}{\mathrm{\delta}}$ & $\begin{array}{l}8 \\
8 \\
8 \\
\end{array}$ & 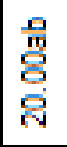 & 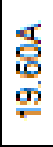 & & 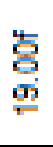 & 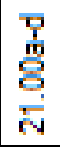 & 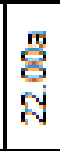 & 蒿 & 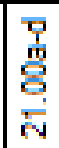 & 名 & \\
\hline & 总 & $>$ & ్ㅜㄹ & 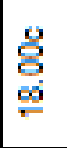 & $\begin{array}{l}8 \\
8 \\
80 \\
0\end{array}$ & $\begin{array}{l}\text { 䎡 } \\
\text { 号 }\end{array}$ & 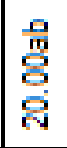 & 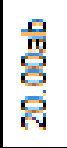 & 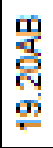 & ్ㅜㄹ & $\begin{array}{l}\text { 点 } \\
\text { क्ष }\end{array}$ & 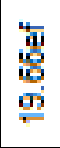 & 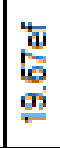 & 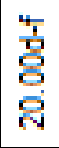 & 范 & 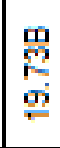 & \\
\hline & 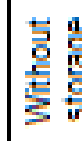 & 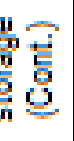 & 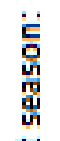 & 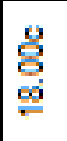 & $\frac{m}{8}$ & 䁶 & $\begin{array}{l}8 \\
8 \\
8 \\
\text { ळ. }\end{array}$ & $\begin{array}{l}8 \\
8 \\
\infty \\
0\end{array}$ & $\begin{array}{l}\text { क्ष } \\
\text { \%े } \\
\text { क्ष }\end{array}$ & 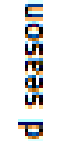 & 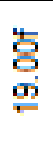 & 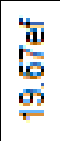 & 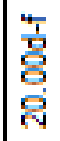 & 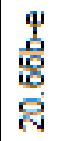 & 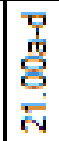 & 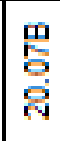 & \\
\hline & & 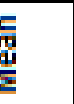 & 实 & 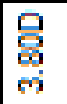 & \begin{tabular}{l} 
罗 \\
\multirow{\forall}{*}{}
\end{tabular} & 疍 & \begin{tabular}{l}
0 \\
\multirow{E}{*}{} \\
$\dot{v}$
\end{tabular} & 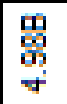 & & $\begin{array}{l}\text { 莒 } \\
\text { 品 }\end{array}$ & 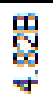 & 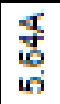 & 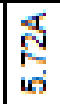 & 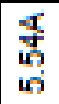 & 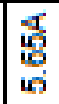 & & \\
\hline & 這 & $I$ & & $\begin{array}{l}\overline{8} \\
\text { 总 } \\
\text { 户 }\end{array}$ & $\begin{array}{l}\text { 罗 } \\
\text { 品 }\end{array}$ & $\begin{array}{l}\text { 롱 } \\
\text { 产 } \\
\text { + }\end{array}$ & 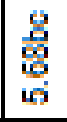 & $\begin{array}{l}\text { 罗 } \\
\text { 品 }\end{array}$ & 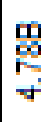 & & 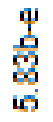 & $\begin{array}{l}\frac{0}{5} \\
\stackrel{8}{0} \\
0\end{array}$ & 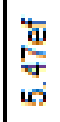 & 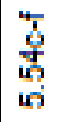 & 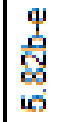 & $\begin{array}{c}\text { 営 } \\
\text { w }\end{array}$ & \\
\hline 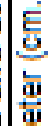 & के & $>$ & & \begin{tabular}{|l}
$E$ \\
N \\
ल
\end{tabular} & 号 & 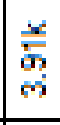 & 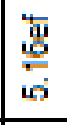 & $\begin{array}{l}\text { 怼 } \\
\text { ஸ }\end{array}$ & 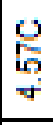 & & $\begin{array}{l}\text { 总 } \\
\text { ल }\end{array}$ & 思 & 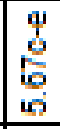 & 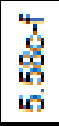 & 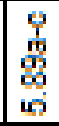 & 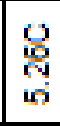 & \\
\hline 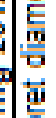 & $\frac{5}{2}$ & $I$ & & $\begin{array}{l}\text { 莺 } \\
\text { ले } \\
\text { 户 }\end{array}$ & $\begin{array}{l}\frac{0}{5} \\
\dot{\sigma} \\
\dot{v}\end{array}$ & 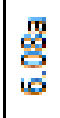 & $\begin{array}{l}\underset{\mathrm{C}}{\mathrm{C}} \\
\text { 品 }\end{array}$ & $\begin{array}{l}\text { 夁 } \\
\dot{v}\end{array}$ & 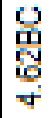 & & $\underset{\mathrm{v}}{\mathrm{N}}$ & 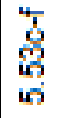 & 总 & $\begin{array}{l}\text { 宗 } \\
\text { 广े }\end{array}$ & 兵 & Oํํำ & \\
\hline & 总 & $>$ & & $\begin{array}{l}E \\
\\
\end{array}$ & $\underset{\mathrm{N}}{\mathrm{N}}$ & ูㅗํ & $\begin{array}{l}\overline{6} \\
\text { ले }\end{array}$ & $\begin{array}{l}\text { 듬 } \\
\text { 品 } \\
\dot{v}\end{array}$ & 응 & & $\begin{array}{l}\text { 哥 } \\
\text { qu }\end{array}$ & 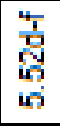 & 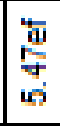 & 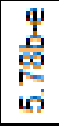 & 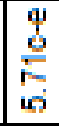 & $\begin{array}{l}\text { 息 } \\
\text { นึ่ }\end{array}$ & \\
\hline & 蒙 & 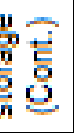 & & 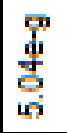 & $\begin{array}{l}\text { 总 } \\
\text { o }\end{array}$ & $\begin{array}{l}8 \\
8 \\
8 \\
\text { ஸे }\end{array}$ & 恕 & $\begin{array}{l}\text { 맹 } \\
\text { 잉 }\end{array}$ & & & 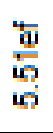 & 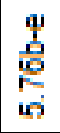 & 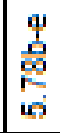 & 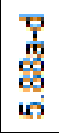 & 今. & $\underset{\text { ณ }}{\stackrel{4}{3}}$ & \\
\hline & 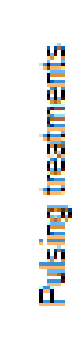 & & & 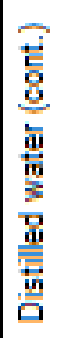 & 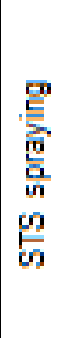 & 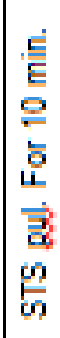 & 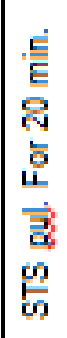 & 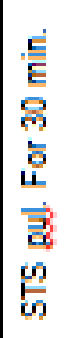 & & & 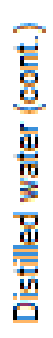 & 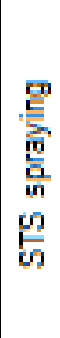 & 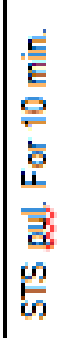 & 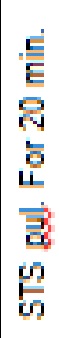 & 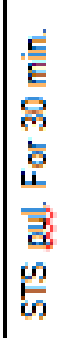 & $\begin{array}{l}\text { 骂 } \\
\text { 恶 }\end{array}$ & \\
\hline
\end{tabular}


photosynthesis were still continuous under storage at room temperature, so cut flowers were supplied with energy-produced metabolites necessary for their freshness, while cold storage prevents depletion of such materials by decreasing the rate of respiration (Celikel and Reid, 2002). In this regard, Shahri et al., (2011) observed that pulsing spikes of Consolida ajacis cv. Violet Blue in $0.5 \mathrm{mM}$ STS solution prior to $72 \mathrm{~h}$ wet storage at $5{ }^{\circ} \mathrm{C}$ and transferring them to holding solution containing Sucrose + HQS significantly improved the postharvest performance of this cut flower.

The interaction treatments also improved the means of these two traits with various significant differences compared to control, but the prevalence in the two seasons was for connecting between pulsing in STS solution for $10 \mathrm{~min}$. and cold horizontal storage for 2 days as this combined treatment elevated the means of such two parameters to the utmost high values in both seasons. This may be ascribed to lumping the beneficial effects of both silver thiosulphate and cold storage as mentioned before.

The previous findings were documented by Hutchinson et al., (2003) who suggested that STS and sucrose can improve tuberose vase life and floret opening through improvement of the water balance. Furthermore, Santos et al., (2012) declared that vase life of Epidendrum ibaguense spikes was increased to 6.5 days when pulsed with $2 \mathrm{mM}$ STS for $30 \mathrm{~min}$. The STS applied before cold storage reduced the rate of flower abscission and improved the ratio between transpiration and water uptake. On the same line, were those results gained by Sashikalal and Ranvir (2003), Singh et al., (2007), Macnish et al., (2008) and Singh et al., (2009) on gladiolus.

\section{3- Fresh and dry weight of spikes and dry weight percentage:}

According to data illustrated in Table (3), it can be concluded that all pulsing treatments induced a significant increment in spike fresh and dry weights compared to the control in both seasons. The mastery in the first season was for pulsing in STS solution for $10 \mathrm{~min}$. treatment, while in the second one was for either pulsing in the same solution for the same time or spraying with it on the foliage, as these two latter treatments gave closely near values with non-significant differences between them in the two seasons.

Regarding the effect of storage treatments on such two parameters, it fluctuated in both seasons. The interactions, on the other hand, in proved the means of these two traits in most cases of the two seasons. The heaviest fresh and dry weights of spikes were achieved in both seasons by joining between pulsing in STS solution for $10 \mathrm{~min}$. and cold storage, horizontally for 2 days, as this combined treatment increased spike fresh weight to 18.05 and $19.15 \mathrm{~g}$ and spike dry weight to 9.87 and $8.46 \mathrm{~g}$ in the $1^{\text {st }}$ and $2^{\text {nd }}$ seasons, respectively.

The percent of dry weight was also affected by the used treatments, as it reached the maximal value in the $1^{\text {st }}$ season by pulsing in STS solution for either 10 or 30 min., while in the $2^{\text {nd }}$ one, STS spraying treatment gave the highest dry weight \%. The cold storage, horizontally for 4 days scored in the two seasons the highest dry weight $\%$ compared to the other storage treatments. Vertical cold storage treatment for 4 days interacted with pulsing in STS solution for $30 \mathrm{~min}$. surpassed all the other sole and combined treatments giving in the $1^{\text {st }}$ season the utmost high dry weight $\%$ at all $(61.06 \%)$, while in the $2^{\text {nd }}$ one, that was achieved by combining between the horizontal cold storage for 4 days and spraying with STS solution (52.96\%).

The aforestated results may be interpretted and discussed as previously mentioned in case of water relations, floret diameter and vase life criteria. However, simillar observations were also detected by Rekha and Shankaraiah (2002) on gladiolus, Jul et al., (2007) on amaryllis, Solgi et al., 


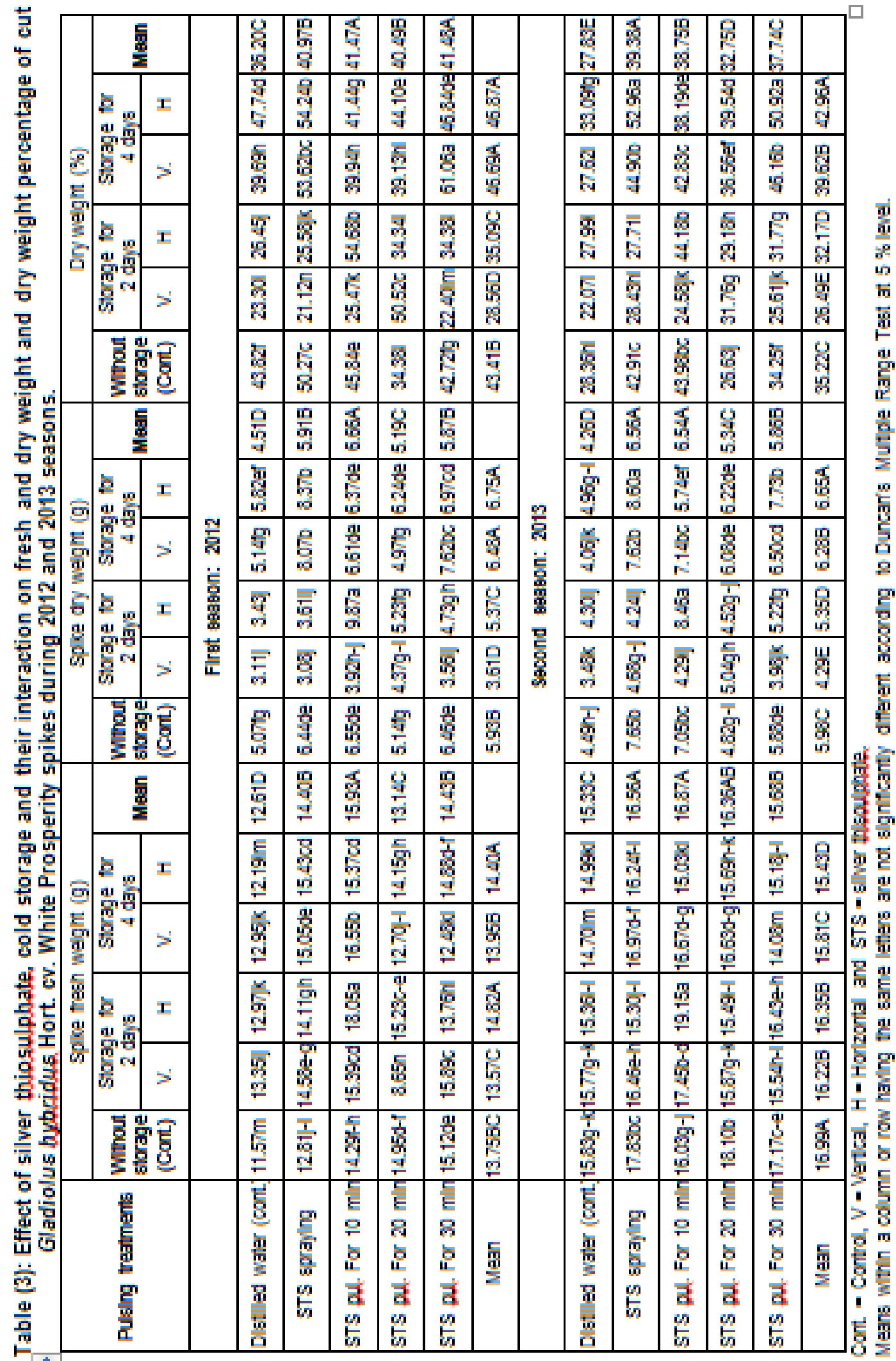


(2009) on gerbera and Hassan and Ali (2014) whom indicated that 1- MCP or STS treatments significantly extended the vase life and minimized the weight loss of gladiolus spikes compared with the control. Both treatments minimized also ethylene production and retained membrane stability. On Asiatic hybrid lily "Orange Tycoon" cut flowering stems, Abd-Allah et al., (2013) noticed that pulsing in STS solution at $1: 4$ $\mathrm{mM}$ for 30 min improved the change \% in fresh weight of inflorescences.

\section{4- Opening and wilting percentage:}

Data in Table (4) exhibit that flower bud opening $\%$ significantly increased in response to the various pulsing treatments employed in this work. However, the highest percent of opening was obtained by pulsing in the STS solution for $10 \mathrm{~min}$. in both seasons. The opposite was right regarding the wilting \%, which decreased significantly by pulsing treatments, with few exceptions in the two seasons. On the other side, opening $\%$ was the maximum in the first season when spikes were kept at room temperature (control), whereas in the second one, that occurred by cold storage treatments, either horizontally for 2 days or vertically for 4 days. Generally, cold storage treatments raised wilting $\%$ with significant difference compared to keeping at room temperature (conted treatment) that reduces the means of such measurement to the least values in the two seasons.

A marked effect on these two parameters was also noticed by the interaction treatments which elevated the percent of opening to the highest averages by combining pulsing in STS solution for 10 min. and cold, horizontal storage for 2 days in both seasons, and diminished the percentage of wilting to the minimal values in the two seasons by combining between pulsing in STS solution for $10 \mathrm{~min}$. and keeping the spikes at room temperature.
This may indicate the role of STS in improving floret opening. In this connection, Serek et al., (1994) affirmed that a climacteric pattern of ethylene production by the youngest buds on the spike of gladiolus (which never opened) was stimulated by cool storage, and was not affected by holding the spikes in a preservative solution containing sucrose. Pulsing the spikes in STS solution improved floret opening but not the life of individual florets. Sucrose and STS had similar but not synergistic effects on floret opening, suggesting that STS improves flower opening in gladiolus by overcoming the effects of carbohydrates depletion. These results, are in harmony with these of Shimizu-Yumoto and Ichimura (2015) on gladiolus cv. "Princess Summer Yellow", Gendy and Hamad (2011) on Strelitzia reginae and Abd-Allah et al., (2013) whom reported that pulsing in STS solution $(1: 4 \mathrm{mM})$ improved flower opening of Asiatic hybrid lily cv. "Orange Tycoon".

\section{5- Leaf and petal chemical composition:}

As shown in Table (5), data reveal that pulsing treatments caused a marked increment in the leaf content of chlorophyll a and $b$ (mg/g f.w.), as well as reducing sugars $\%$ in the leaves and petals with the superiority of pulsing for $10 \mathrm{~min}$. treatment, which recorded the highest means of the aforenamed constituents relative to control (distilled water) and other pulsing treatments. The opposite was right concerning carotenoids content in the leaves and total sugars \% in the leaves and petals, as they decreased as a result of applying different pulsing treatments except of pulsing for $20 \mathrm{~min}$ and for 30 min treatments that slightly increased total sugars content in the leaves and petals over control to 2.03 and $2.67 \%$, respectively.

It was also noticed that content of chlorophyll a and carotenoids in the leaves increased, while that of chlorophyll $b$ declined by cold storage treatments, with the 


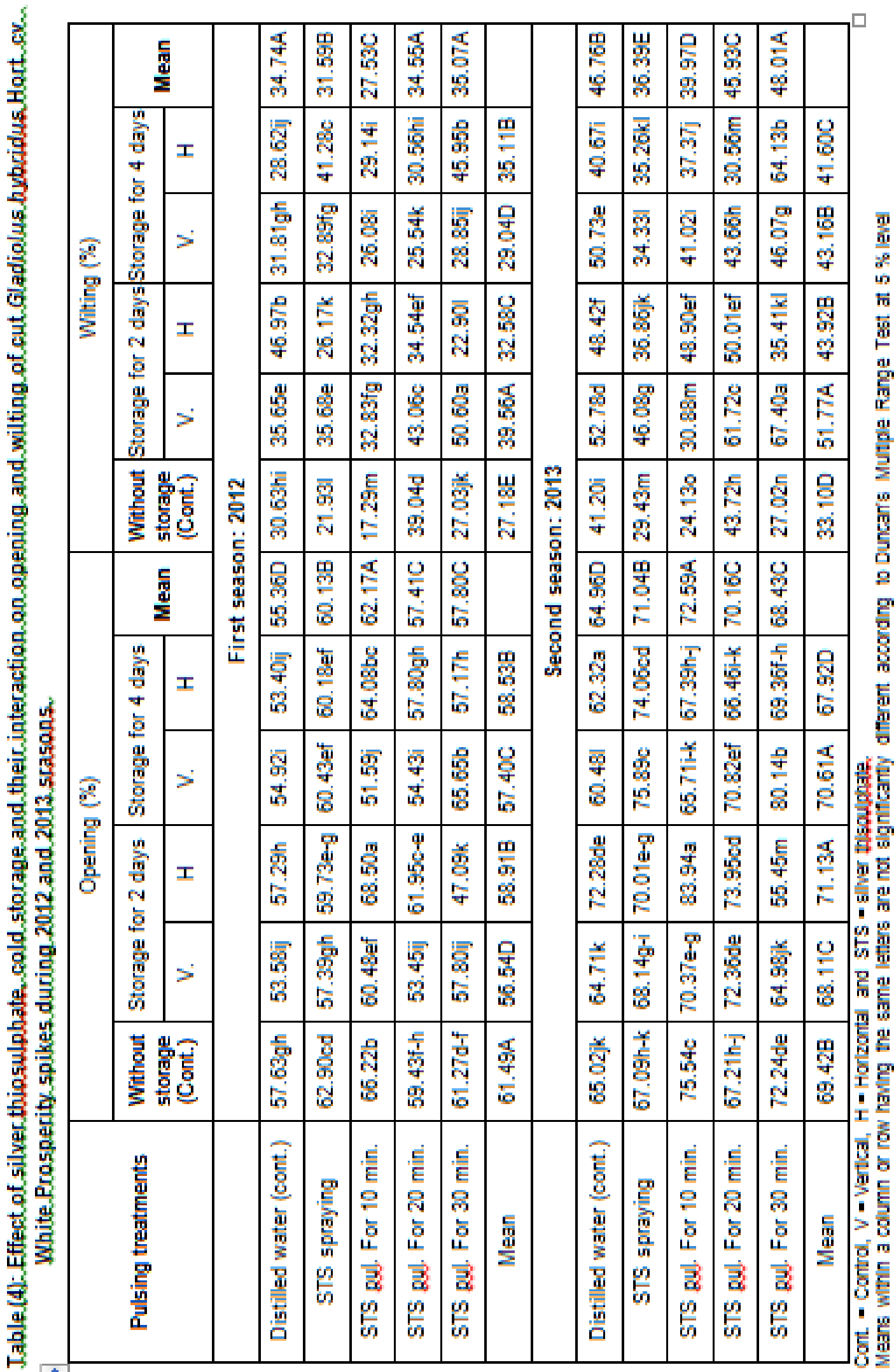


superiority of horizontal storage for 2 days which gave the best pigments content. The percent of total and reducing sugars in the leaves fluctuated, but in petals, data indicated that total sugars \% increased, while reducing sugars $\%$ decreased by the various cold storage treatments compared to storage at room temperature. However, combining between pulsing in STS solution for $10 \mathrm{~min}$. and vertical cold storage for 2 days gave the highest content in most constituents mentioned above.

Table (5): Effect of silver thiosulphate, cold storage and their interaction on leaf and petal chemical composition of cut Gladiolus hybridus Hort. cv. White Prosperity spikes during 2013 season.

\begin{tabular}{|c|c|c|c|c|c|c|c|c|}
\hline \multirow{3}{*}{$\begin{array}{c}\text { Cold } \\
\text { storage } \\
\text { treatments }\end{array}$} & \multirow{3}{*}{$\begin{array}{l}\text { Under the room } \\
\text { conditions (in the Lab) }\end{array}$} & \multicolumn{3}{|c|}{ Pigments content (mg/g f.w.) } & \multirow{2}{*}{\multicolumn{2}{|c|}{$\begin{array}{c}\text { Total sugars } \\
(\%)\end{array}$}} & \multirow{2}{*}{\multicolumn{2}{|c|}{$\begin{array}{l}\text { Reducing } \\
\text { sugars (\%) }\end{array}$}} \\
\hline & & \multirow{2}{*}{ Chlorophyll a } & \multirow{2}{*}{ Chlorophyll b } & \multirow{2}{*}{ Carotenoids } & & & & \\
\hline & & & & & Leaves & Petals & Leaves & Petals \\
\hline \multirow{5}{*}{$\begin{array}{c}\text { Without } \\
\text { storage } \\
\text { (cont.) }\end{array}$} & Distilled water (cont.) & 0.84 & 0.50 & 0.44 & 1.47 & 0.33 & 1.04 & 1.99 \\
\hline & STS spraying & 2.78 & 1.93 & 1.13 & 1.52 & 0.56 & 1.57 & 2.03 \\
\hline & STS pul. For $10 \mathrm{~min}$. & 3.23 & 3.35 & 1.17 & 1.82 & 2.71 & 1.70 & 2.54 \\
\hline & STS pul. For $20 \mathrm{~min}$. & 2.84 & 2.23 & 0.73 & 1.47 & 3.35 & 1.04 & 2.29 \\
\hline & STS pul. For 30 min. & 2.08 & 2.93 & 0.60 & 1.55 & 2.61 & 0.99 & 2.35 \\
\hline & Mean & 2.35 & 2.19 & 0.81 & 1.57 & 1.37 & 1.27 & 2.24 \\
\hline \multirow{12}{*}{$\begin{array}{l}\text { Storage } \\
\text { for } 2 \text { days }\end{array}$} & \multirow{2}{*}{$\begin{array}{c}\text { Distilled water (cont.) } \\
\text { STS spraying }\end{array}$} & 1.35 & 0.05 & 0.59 & 1.34 & 1.23 & 1.12 & 1.07 \\
\hline & & 2.64 & 2.73 & 0.71 & 1.31 & 1.66 & 1.21 & 1.56 \\
\hline & V STS pul. For $10 \mathrm{~min}$. & 2.27 & 2.83 & 3.61 & 2.46 & 3.82 & 2.36 & 3.61 \\
\hline & STS pul. For $20 \mathrm{~min}$. & 1.92 & 1.01 & 2.31 & 1.86 & 0.56 & 1.34 & 1.41 \\
\hline & STS pul. For $30 \mathrm{~min}$. & 1.69 & 1.44 & 0.52 & 1.23 & 1.97 & 0.97 & 1.94 \\
\hline & Mean & 1.97 & 1.61 & 1.55 & 1.64 & 1.85 & 1.40 & 1.92 \\
\hline & \multirow{2}{*}{$\begin{array}{c}\text { Distilled water (cont.) } \\
\text { STS spraying }\end{array}$} & 0.91 & 1.87 & 1.60 & 0.88 & 0.94 & 0.45 & 1.73 \\
\hline & & 4.98 & 2.25 & 1.93 & 1.78 & 3.06 & 0.58 & 0.94 \\
\hline & STS pul. For $10 \mathrm{~min}$. & 5.26 & 4.07 & 3.21 & 2.36 & 2.23 & 1.81 & 3.00 \\
\hline & STS pul. For $20 \mathrm{~min}$. & 1.86 & 1.88 & 1.82 & 1.04 & 1.08 & 0.58 & 0.98 \\
\hline & STS pul. For $30 \mathrm{~min}$. & 1.60 & 1.88 & 2.03 & 1.48 & 1.93 & 0.72 & 1.91 \\
\hline & Mean & 2.92 & 2.39 & 2.12 & 1.51 & 1.85 & 0.83 & 1.71 \\
\hline \multirow{12}{*}{$\begin{array}{l}\text { Storage } \\
\text { for } 4 \text { days }\end{array}$} & \multirow{2}{*}{$\begin{array}{c}\text { Distilled water (cont.) } \\
\text { STS spraying }\end{array}$} & 2.25 & 0.74 & 1.13 & 1.06 & 0.85 & 0.45 & 0.34 \\
\hline & & 2.61 & 1.27 & 2.54 & 1.22 & 3.12 & 0.60 & 2.24 \\
\hline & V STS pul. For $10 \mathrm{~min}$. & 2.37 & 2.46 & 3.94 & 1.73 & 1.38 & 1.17 & 1.58 \\
\hline & STS pul. For $20 \mathrm{~min}$. & 3.05 & 2.45 & 1.38 & 1.16 & 2.02 & 2.22 & 1.26 \\
\hline & STS pul. For $30 \mathrm{~min}$. & 3.65 & 1.45 & 1.53 & 1.17 & 1.80 & 0.86 & 1.33 \\
\hline & Mean & 2.79 & 1.67 & 2.10 & 1.27 & 1.83 & 1.06 & 1.35 \\
\hline & \multirow{2}{*}{$\begin{array}{c}\text { Distilled water (cont.) } \\
\text { STS spraying }\end{array}$} & 1.64 & 1.41 & 0.33 & 1.06 & 0.65 & 1.62 & 1.24 \\
\hline & & 1.41 & 1.33 & 1.21 & 1.91 & 1.57 & 1.77 & 1.39 \\
\hline & $\mathrm{H}$ STS pul. For $10 \mathrm{~min}$. & 7.49 & 2.30 & 1.26 & 2.45 & 2.81 & 1.89 & 2.59 \\
\hline & STS pul. For $20 \mathrm{~min}$. & 1.44 & 1.06 & 0.45 & 2.60 & 2.20 & 1.85 & 1.96 \\
\hline & STS pul. For $30 \mathrm{~min}$. & 4.43 & 1.10 & 2.00 & 1.77 & 3.20 & 1.75 & 3.04 \\
\hline & Mean & 3.28 & 1.44 & 1.05 & 1.96 & 2.09 & 1.78 & 2.04 \\
\hline \multirow{5}{*}{ Mean } & Distilled water (cont.) & 1.40 & 0.91 & 0.82 & 1.16 & 0.80 & 0.94 & 1.27 \\
\hline & STS spraying & 2.88 & 1.90 & 1.50 & 1.55 & 1.99 & 1.15 & 1.63 \\
\hline & STS pul. For $10 \mathrm{~min}$. & 4.12 & 3.00 & 2.64 & 2.16 & 2.59 & 1.79 & 2.66 \\
\hline & STS pul. For 20 min. & 2.22 & 1.73 & 1.34 & 1.63 & 1.84 & 1.41 & 1.58 \\
\hline & STS pul. For $30 \mathrm{~min}$. & 2.69 & 1.76 & 1.34 & 1.44 & 2.30 & 1.06 & 2.11 \\
\hline
\end{tabular}

Cont. = Control, $\mathrm{V}=$ Vertical, $\mathrm{H}=$ Horizontal and STS = silver thisoulphate. 
This may indicate the role of both STS and cold storage in the presence of sucrose in preventing depletion of active constituents in spike tissues. In this regard, Hassan and Ali (2014) found that silver thiosulphate at 0.2 or $0.4 \mathrm{mM}$ for $6 \mathrm{~h}$. enhanced the relative water content in gladiolus spikes and maintained chlorophyll and carbohydrates contents compared with the control spikes which were kept in distilled water. On Birdof-Paradise, Gendy and Hamad (2011) pointed out that pulsing treatment of STS at 1: $4 \mathrm{mM}$ for 30 min maintained anthocyanin content in petals. The present results confirm the previous reports mentioned by Singh et al., (2007) and Marandi et al., (2011) on gladiolus and Bayleyegn et al., (2012) who postulated that passive refrigeration system and pulse with mixtures of STS, chrysal clear solution and 8-HQS maintained a fresh-like quality of rose flowers and TSS contents of petals.

From the previous findings, it can be advised to pulse the cut spikes of gladiolus cv. "White Prosperity" with STS solution for $10 \mathrm{~min}$. and then preserving them in $10 \%$ sucrose solution at room temperature for local marketing, or storage them after pulsing, horizontally for at least 2 days at 5 ${ }^{\circ} \mathrm{C}$ for shipping abroad.

\section{REFERENCES}

Abd-Allah, A. A., Mona A. Darwish, Samia S. Helme, Soad A. M. Khenizy and Reham, E. A. Alm-Eldeen (2013). Response of Asiatic hybrid lily cv. Orange Tycoon cut flowering stems to some pulsing and holding solutions, storage temperature and their interactions. J. Hort.Sci. \& Ornam. Plants, 5 (3):202-217.

Abdel-Moniem, Azza, M., Ola, A. Amin and Gehan H. Abdel-fattah (2012). Effect of some preservative solutions on vase life and quality of three rose (Rose hybrida L.) cultivars. J. Biol. Chem. \& Environ. Sci., 7 (4): 537-555.

Bai, J.G., P.L. Xu, C. S. Zong and C.Y. Wang (2009). Effects of exogenous calcium on some postharvest characteristics of cut gladiolus. Agric. Sci. China, 8: 293-303.

Bailey, L. H. (1976). Hortus Third, Macmillan Publishing Co., Inc., 866 Third Avenue, New york, N.Y. 10022. Printed in USA, pp. 1290.

Bayleyegn, A., B. Tesfaye and T.S. Workneh (2012). Effects of pulsing solution, packaging material and passive refrigeration storage system on vase life and quality of cut rose flowers. African J. Biotech., 11 (16): 3800-3809.

Beura, S., S. Ranvir, S. Beura and R. Singh (2001). Effect of pulsing before storage on postharvest life of gladiolus. J. Ornam. Hort., 4: 91-94.

Celikel, F.G., J. C. Cevallos and M. S. Reid (2010). Temperature, ethylene and the postharvest performance of cut snapdragons (Antirrhinum majus). Scientia Hort., 125: 429-433.

Celikel, F. G. and M. S. Reid (2002). Storage temperature affects the quality of cut flowers from the Asteraceae. HortScience, 37 (1): 148-150.

Dubois, M., F. Smith, K. A. Illes, J. K. Hamilton and P. A. Rebers (1966). Colorimetric mehod for determination of sugars and related substances. Ann. Chem., 28 (3): 350-356.

Gendy, A. S. and E. H. Hamad (2011). Effect of some storage and preservative solution treatments on vase life and quality of Strelitzia reginae L. cut flowers. J. product. \& Dev. 16 (3): 397-414.

Han, Susan, S. (2001). Factors affecting postharvest quality of cut lilies. Crop Cultivation, GPN, September: 29-33.

Hassan, F. (2005). Postharvest studies on some important flower crops. Doctoral Thesis, Corvinus Univ. of Budapest, Budapest, Hungary.

Hassan, F.A.S. and E. Ali (2014). Physiological response of gladiolus flowers to anti ethylene treatments and their relation to senescence. Inter. J. Adv. Res., 2 (10): 188-199. 
Hutchinson, M. J., D. K. Chebet and V. E. Emongor (2003). Effect of Accel, sucrose and silver thiosulphate on the water relations and postharvest physiology of cut tuberose flowers. African Crop Sci. J., 11(4): 279- 287.

Jul, F., I. Tahir and S.M. Sultan (2007). Effect of storage temperature on postharvest performance of Amaryllis belladonna L. CV. Rosa scapes. J. Plant Boil., 34: 43-47.

Marandi, R. J., A. Hassani, A. Abdo-llahi and S. Hanafi (2011). Improvement of the vase life of cut gladiolus flowers by essential oils, salicylic acid and silver thiosulphate. J. Medicinal Plants Res., 5 (20): 5039-5043.

Mead, R., R. N. Curnow and A. M. Harted (1993). Statistical Methods in Agriculture and Experimental Biology. $2^{\text {nd }}$ Ed., Chapman \& Hall Ltd., London, 335 pp.

Moran, R. (1982). Formula for determination of chlorophyllous pigment extracted with $\mathrm{N}-\mathrm{N}$-dimethyl formamide. Plant Physiol, 69: 1376-81.

Muchiri, J. (2005). Effects of chemical pretreatments, cold storage and water quality on the postharvest quality of Eustoma grandiflorum L. cut flowers. Univ. of Nairobi. http://erepository.uonbi.ac.ke.:8080/xmlul / handle/

Philosoph-Hadas, S., S. Meir, I. Rosenberger and A.H. Halevy (1996). Regulation of the gravitropic response and ethylene biosynthesis in gravistimulated snapdragon spikes by calcium chelators and ethylene inhibitors. Plant Physiol., 110: 301-310.

Raid, M. S., J. L. Paul, M.B. Farhoomand, A.M. Kofranek and G. L. Staby (1980 b). Pulse treatments with the silver thiosulphate complex extend the vase life of cut carnations. J. Amer. Soc. Hort. Sci., 105 (1): 25-27.

Rekha, M. K. and V. Shankaraiah (2002). Effect of storage conditions and preservative solutions on vase life of cut gladiolus spikes. Proceedings of the
National Symposium on Indian Floriculture in the New Millennium, Lal Bagh, Bangalore, 25-27 Feb., pp. 126129.

Santos, J. S., F. L. Finger, Mapeli, M. Ana, J. Karsten, Teresa, D.C. Mendes and J. G. barbosa (2012). Longevity of Epidendrum ibaguense inflorescences influenced by the storage temperature and ethylene inhibitor. Braz. Arch. Biol. Technol., 55 (2): 1-8.

SAS Institute (2009). SAS/STAT User's Guides Statistics. Vers. 9.0, SAS. Institute Inc. Cary, N.C., USA.

Sashikalal, B. and S. Ranvir (2003). Effect of storage temperature and warpping material on postharvest life of gladiolus cv. Her Majesty. J. Ornam. Hort., 6 (4): 322-327.

Serek, M., R.B. Jones and M.S. Reid (1994). Role of ethylene in opening and senescence of Gladiolus sp. flowers. J. Amer. Soc. Hort. Sci., 119 (5): 10141019.

Shahri, W., I. Tahir, S. T. Islam and M. A. Bhat (2011). Synergistic effect of STS and cool storage on postharvest performance of cut spikes of Consolida ajacis cv. Violet Blue. Hort. Environ. Biotech., 52 (5): 466-470.

Shimizu-Yumoto, H. and K. Ichimura (2015). Effect of storage in packaging with oxygen absorbers on the quality of cut gladiolus "Princess Summer Yellow" spikes. Postharvest Biol. \& Tech., 111: 191-196.

Singh, A., J. Kumar and P. Kumar (2007). Effect of different packaging films and cold storage durations on keeping quality of gladiolus cut spikes. J. Ornam. Hort., 10 (4): 235-239.

Singh, A., J. Kumar and P. Kumar (2009). Influence of sucrose pulsing and sucrose in vase solution on flower quality of modified atmosphere low temperature (MALT)-stored gladiolus cut spikes. IX Inter. Sym. on Postharvest Quality of Ornam. Plants, Odense, Denmark, 11-14 Aug., pp. 129-138. 
Solgi, M., M. Kafi, T.S. Taghavi and R. Naderi (2009). Essential oil and silver nanoparticles as novel agents to extend vase life of gerbera (Gerbera jamesonii cv. Dune) flowers. Postharvest Biol. Tech., 53: 217-226.

Steel, R. G. D. and J. H. Torrie (1980). Principles and procedures of statistics. McGrow Hill Book Co., Inc., New York, pp: 377-400.

Watkins, C. B. and Miller, W.B. (2005). A summary of physiological processes or disorders in fruits, vegetables and ornamental products that are delayed or decreased, increased, or unaffected by 1- methylcyclopropane. http:/www.hort.cornell.edu./Watkins/ethyl ene/index.htm.

Yamada, T., Y. Takatsu, T. Manabe, M. Kasumi and W. Marubashi (2003). Supperssive effect of trehalose on apoptotic cell death leading to petal senescence in ethylene-insensitive flowers of gladiolus. Plant Sci., 169: 213221. 


\title{
تحسين عمر وجودة نورات الجلاديولس المقطوفة (صنف بروسبرتى الأبيض) بمثبط الإيثلين، التخزين البارد والحفظ فى محلول سكروز
}

\author{
عزة محمد عبد المنعم \\ قسم بحوث الزينة وتتسيق الحدائق، معهد بحوث البساتين، مركز البحوث الزراعية، الجيزة، مصر.
}

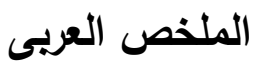

أجريت هذه الدراسة بمعمل معاملات ما بعد القطف بقسم بحوث الزينة، معهد بحوث البساتين، الجيزة، مصر

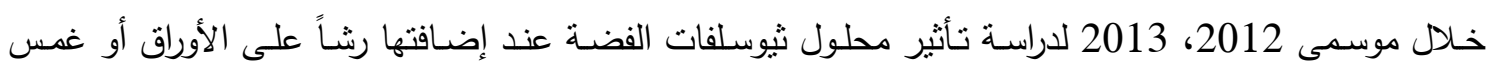

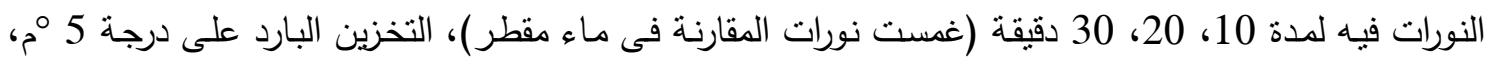
رأسيا أو أفقياً لمدة يومين أو أربعة أيام (بجانب معاملة المقارنة والتى حفظت فيها النورات على درجة حرارة الغرفة النة

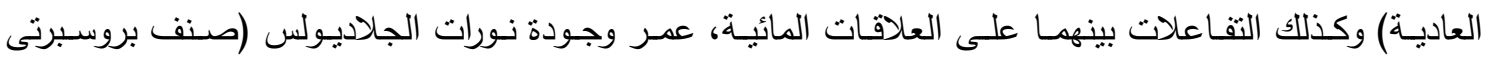
الأبيض) بعد القطف. عقب تطبيق كل معاملة من المعاملات السابقة حفظت قواعد النورات فى محلول سكروز

أوضحت النتائج المتحصل عليها أن معاملات ثيوسلفات الفضـة أحدثت زيادة معنوية فى كمية الماء الممتصـة بواسطة نورات الجلاديولس، لكنها على النقيض قللت كمية المياه المفقودة من هذه النورات مقارنة بالكنترول فى لي معظم الحالات بكلا الموسمين. أدت هذه المعاملات أيضاً إلى تحسين قطر الزهيرات، عمر النورات فى الفازة،

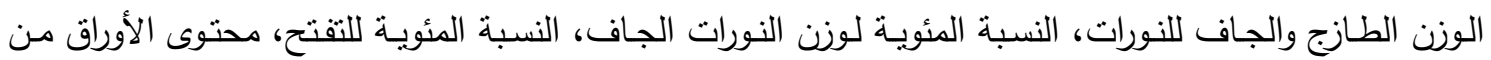
كلورفيللى أ، ب وكذللك محتوى الأوراق والبتلات من السكريات المختزلة، بينما قللت نسبة الذبول، محتوى الأوراق

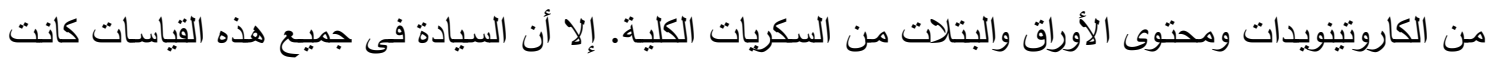

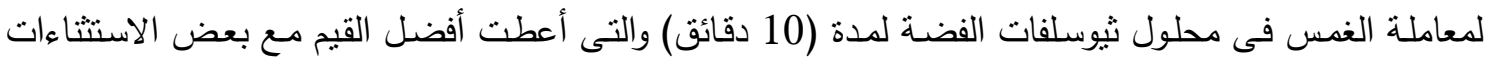

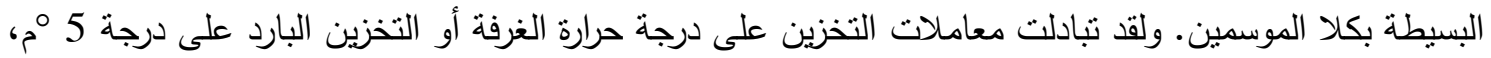
رأسياً أو أفقياً لمدة يومين أو أربعة أيام تحسين متوسطات الصفات السابقة، مع تفوق معاملة التخزين البارد أفقياً

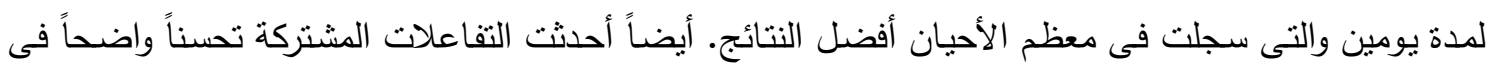
معظم القياسـات، لكن السيادة كانت لمعاملـة الجمـع بين الغمس فـى محلول ثيوسـلفات الفضــة لمدة 10 دقائق

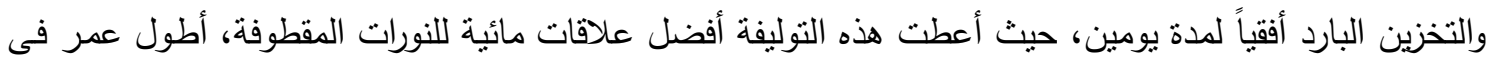
الفازة واعلى جودة مقارنة بالمعاملات الفردية والمشتركة الأخرى. وعليه، يمكن التوصية بغدس نورات الجلاديولس المقطوفة (صنف بروسبرتى الأبيض) فى محلول ثيوسلفات

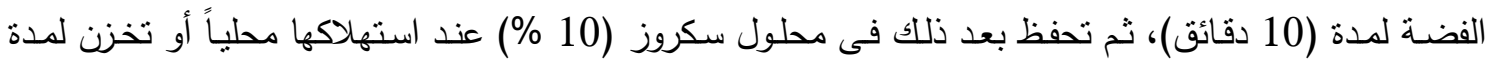
يومين على الأقل أفقياً على درجة (5 مجاتم) عند شحنها للخارج. 
